\title{
Photoionization models of roughly circular Galactic planetary nebulae in the thick disk ${ }^{\star}$
}

\author{
M. Emprechtinger ${ }^{1}$, T. Rauch ${ }^{2,3}$, and S. Kimeswenger ${ }^{1}$ \\ 1 Institut für Astrophysik der Universität Innsbruck, Technikerst. 25, 6020 Innsbruck, Austria \\ e-mail: martin . emprechtinger@uibk.ac .at \\ 2 Dr.-Remeis-Sternwarte, Sternwartstraße 7, 96049 Bamberg, Germany \\ ${ }^{3}$ Institut für Astronomie und Astrophysik, Abteilung Astronomie, Sand 1, 72076 Tübingen, Germany
}

Received 7 July 2004 / Accepted 6 October 2004

\begin{abstract}
We present the result of photo-ionizing modelling of the three planetary nebulae (PNe) A 20, A 15 and MeWe 1-3. All three objects are roughly circular, highly excited and have a high Galactic $z$. The PNe display low densities in the shell, but relatively dense halos. A low metallicity and a relative high electron temperature are found. Comparisons with radio observations confirm the obtained properties. The objects very likely originate from thick disk stellar progenitors. The distances found investigating the PNe shells are somewhat lower than those derived spectroscopically for the central stars in the past.
\end{abstract}

Key words. ISM: planetary nebulae: individual: A 20 - ISM: planetary nebulae: individual: A 15 - ISM: planetary nebulae: individual: MeWe 1-3

\section{Introduction}

Although the interacting wind model for the formation of planetary nebulae (PNe) has been accepted for a long time (Kwok et al. 1978), the exact mechanism is not yet clearly understood up to now. Several numerical simulations have been carried out especially during the last decade (e.g. Frank \& Mellema 1994a; Dwarkadas \& Balick 1998; or Schönberner \& Steffen (2002). The latter carried out a one-dimensional hydrodynamic simulation based on the evolutionary tracks of Blöcker (1995) and the simulations of the circumstellar shell around Asymptotic Giant Branch (AGB) stars of Steffen et al. (1998).

The results of their simulation showed that a shockbounded ionized main PN shell moving supersonically into the AGB-material compresses the inner parts of the matter into a dense but thin shell. The unaffected AGB-material becomes ionized as well and forms a rapidly expanding halo. Because of the drop of luminosity of the central star of the PN (CSPN) towards the white dwarf regime the outer part of the PN shell recombines quickly forming an second inner halo. The disadvantage of this simulation is that it just considers one dimension. Thus more complex interactions of the winds such as Rayleigh-Taylor instabilities, which quickly build a clumpy environment as we see in HST images, are not taken into account. For this reason the filling factor $(\varepsilon)$ was set to unity. Comparing

* Based on observations collected at the European Southern Observatory, La Silla under prog-id. 56.D-0717 and on observations made with the European Southern Observatory telescopes obtained from the ESO/ST-ECF Science Archive Facility. densities obtained from forbidden line ratios and those obtained from radio data or similar methods clearly shows a filling factor far below unity (e.g. Mallik \& Peimbert 1988; Boffi \& Stanghellini 1994). This changes dramatically the optical thickness to ionizing UV photons and thus a recombining PN-shell is doubtful (Armsdorfer et al. 2002).

The three PNe A 20, A 15 (Abell 1955) and MeWe 1-3 (Melmer \& Weinberger 1990) were selected because they are roughly circular. The central stars of the PNe (CSPNe) have been well studied spectroscopically (Rauch et al. 1999; McCarthy et al. 1997; Saurer et al. 1997). This allows us to fix the input of radiation for the nebula. We have modelled the physical properties of the PNe-shells of the three objects and compared the results with the theory.

\section{Input data}

\subsection{Observations and data reduction}

Most data were obtained on January 29 and 30, 1992 using the New Technology Telescope (NTT) with the EMMI spectrograph at ESO, La Silla, Chile and were taken from the ESO data archive. THX31156 (red arm) and TK1024AF (blue arm) CCDs, and grating \#8 were used. The slit-width was adjusted to 1 '.'2. The spatial resolution was 0.44 per pixel at the red arm of the instrument (480 nm to $680 \mathrm{~nm}$ ) and 0.37 at the blue arm $(400 \mathrm{~nm}$ to $505 \mathrm{~nm})$. Additionally, A 20 was observed by one of us (TR) in 1996 February 10th with the ESO $3.60 \mathrm{~m}$ telescope using the EFOSC1 spectrograph and the \#26 TEK512M 1215-3 detector at a resolution of 0.' 61 per pixel 
Table 1. Basic data for the PNe sample.

\begin{tabular}{lcccc}
\hline \hline Name & $\begin{array}{c}\text { GPN } \\
\text { (Kimeswenger 2001) }\end{array}$ & $\begin{array}{c}F_{1.4 \mathrm{GHz}} \\
{[\mathrm{mJy}]}\end{array}$ & IRAS \\
\hline A 20 & G214.96+07.81 & 10.4 & $07203+0151$ \\
A 15 & G233.53-16.31 & $<2.5$ & $06249-2520$ \\
MeWe 1-3 & G308.26+07.79 & - & $13249-5426$ \\
\hline
\end{tabular}

Table 2. Interstellar foreground extinction from the Balmer decrement as derived here and taken from literature.

\begin{tabular}{lcc}
\hline \hline Name & $\begin{array}{c}E(B-V) \\
\text { here }\end{array}$ & $\begin{array}{c}E(B-V) \\
\text { Tylenda et al. (1992) }\end{array}$ \\
\hline A 15 & $0^{\mathrm{m}} 00$ & $0.04-0.64$ \\
A 20 & $0^{\mathrm{m}} 05$ & 0. m $30-0.35$ \\
MeWe 1-3 & 0.927 & - \\
\hline
\end{tabular}

(from $380 \mathrm{~nm}$ to $530 \mathrm{~nm}$ ). At EFOSC narrow-band observations using [O III] and $\mathrm{H} \alpha$ filters were carried out too.

The calibration was done following the usual procedure in MIDAS and the calibration data from the EMMI and EFOSC1 manuals. The standard star EG 21 (Hamuy et al. 1992) was used for flux calibration.

The target selection for this investigation used two criteria: first, the PN should have a roughly circular shape; second, the CSPN should have been studied spectroscopically.

For all three objects IRAS counterparts were found and radio observations at $1.4 \mathrm{GHz}$ have been done for two of them (Condon \& Kaplan 1998). The objects are summarized in Table 1.

\subsection{Spectroscopy}

All three objects are characterized by an intensive HeII line $(\lambda=468.3 \mathrm{~nm})$ and a total absence of [NII] and [SII] lines. The [OIII] lines at $\lambda=495.9 \mathrm{~nm}$ and $\lambda=500.7 \mathrm{~nm}$ are also much weaker than expected. The extinction was derived from the Balmer decrement, and to deredden the frames we used the interstellar extinction law by Savage \& Mathis (1979). The results are given in Table 2. For A15 and A20 our results from the Balmer decrement of the high $\mathrm{S} / \mathrm{N}$ spectra differ significantly from those derived from the survey spectra in Tylenda et al. (1992). After dereddening with our values from Table 2 the continuum of the CSPN fits our results perfectly. Also the $B-V$ of the CSPN from the literature supports our results. The resulting $(B-V)_{0}$ is then always between -0.34 and -0 . 25 . This is expected for the hot CSPNe. The relative errors of the line ratios were estimated by the variations of the ratios of $\mathrm{H} \alpha / \mathrm{H} \beta$ and $[\mathrm{OIII}]_{500.7} /[\mathrm{OIII}]_{495.9}$ along the radius. A conservative estimation gives an error of 10-15\% for the relative line strength. Since the three [OIII] lines at $\lambda=500.7 \mathrm{~nm}, \lambda=495.9 \mathrm{~nm} \&$ $\lambda=436.3 \mathrm{~nm}$ have been detected, a temperature determination was possible (Table 3 ). The error of the ratio of [OIII] lines was assumed to be $50 \%$, because $[\mathrm{OIII}]_{436.3}$ was very faint. Hence the accuracy of $T_{\mathrm{e}}$ is about $T_{-3000}^{+11000} \mathrm{~K}$. Although the error of $T_{\mathrm{e}}$ is relative high, it turned out that the electron temperature
Table 3. Electron temperatures derived from [OIII] lines and computed in CLOUDY:

\begin{tabular}{lccc}
\hline \hline Name & $\frac{I_{495.9}+I_{500.7}}{I_{436.3}}$ & $T_{\mathrm{e}}[\mathrm{K}]$ & $\left\langle T_{\mathrm{e}}^{\text {CLOUDY }}\right\rangle[\mathrm{K}]$ \\
\hline A 15 & 54.7 & $16800_{-3000}^{+11000}$ & 23000 \\
A 20 & 71.5 & $14800_{-3000}^{+11000}$ & 20000 \\
MeWe 1-3 & 62.2 & $15800_{-3000}^{+11000}$ & 22500 \\
\hline
\end{tabular}

Table 4.

\begin{tabular}{lcccc}
\hline \hline Name & $\begin{array}{c}T_{\text {eff }} \\
{[\mathrm{kK}]}\end{array}$ & $\begin{array}{c}L \\
{\left[L_{\odot}\right]}\end{array}$ & $\begin{array}{c}\log g \\
{\left[\mathrm{~cm} / \mathrm{s}^{2}\right]}\end{array}$ & $\begin{array}{c}D_{\mathrm{CSPN}} \\
{[\mathrm{kpc}]}\end{array}$ \\
\hline A 20 & $119 \pm 22$ & 2100 & 6.13 & $4.1 \pm 50 \%$ \\
A 15 & $110 \pm 10$ & 4238 & 5.7 & $4.5 \pm 25 \%$ \\
MeWe 1-3 & $100 \pm 10$ & 4580 & 5.5 & $6.9 \pm 50 \%$ \\
\hline
\end{tabular}

is significantly higher than the $10000 \mathrm{~K}$ typical for PNe. This is a signature of the low metallicity (see below), causing the cooling by forbidden lines to be less effective.

\subsection{Central stars}

The central stars of the objects have been studied extensively well in the past. Detailed spectroscopy was carried out by Rauch et al. (1999) (A 20), McCarthy et al. (1997) (A 15) and Saurer et al. (1997) (MeWe 1-3). To determine distances $D_{\text {CSPN }}$ the method of Heber et al. (1984) was used for A 20 and A 15 in the papers above. In the case of MeWe 1-3 no distance has been published before. We derived it using an Eddington flux $H_{v}$ given by Rauch (2004) following the method of Heber et al. (1984), who gave calibrations for Johnson and Strömgren filters.

Unfortunately no reliable photometry is available for the CSPNe of A 20 and MeWe 1-3, thus the error is considerably higher. But also for A 15 an error of $25 \%$ was assumed following Mendez et al. (1988).

\section{Photoionisation model}

To model the PNe the CLOUDY code (Ferland 1996) was used. As input parameters different values of the radial density profile, the chemical abundances and the stellar properties (within the ranges of error given above) were used. The filling factor was assumed to be 0.1 , which is typical for evolved $\mathrm{PNe}$ (Mallik \& Peimbert 1988; and Boffi \& Stanghellini 1994). The NLTE central star models of Rauch $(1997,2003)$ were used to obtain a proper ionizing spectrum. Armsdorfer et al. (2002) showed the need of real NLTE stellar atmospheres for a good description especially of the HeII lines.

The results give the fraction of ionization of hydrogen and helium as a function of radius, and a mean electron temperature. To obtain the surface brightness, the emissivity was integrated along the line of sight. To do this spherical symmetry was adopted, which is indicated by the round shape of the objects (see Soker 1997; Tylenda et al. 2003, for A 20 and A 15). The surface-brightness in various emission lines has been 

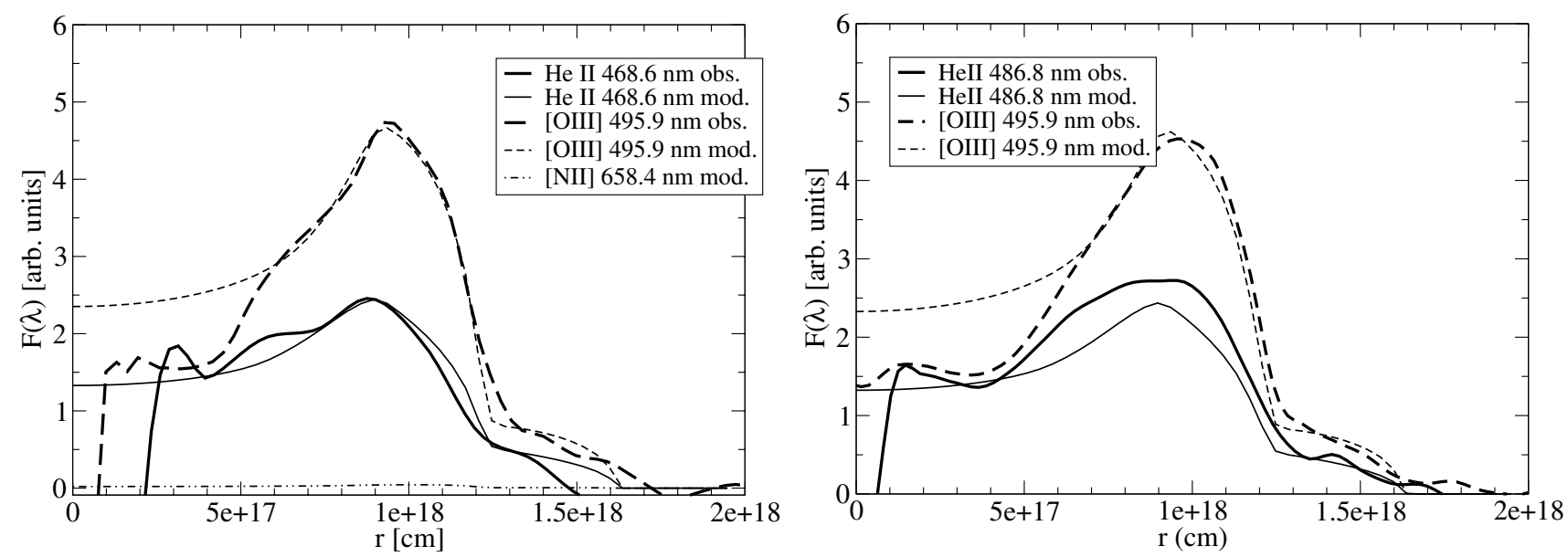

Fig. 1. Observations and model for several lines of A 20. Left: observation with NTT/EMMI (slit N-S); Right: observation with ESO $3.6 \mathrm{~m} / \mathrm{EFOSC}$ (slit E-W); especially in the [OIII] lines the radial symmetry of the object is pronounced. Also the fragmentation into three parts, halo, PN shell and central cavity, can be clearly seen. The [NII] lines are an upper limit and thus confirm clearly the low metallicity found here (see Table 5).
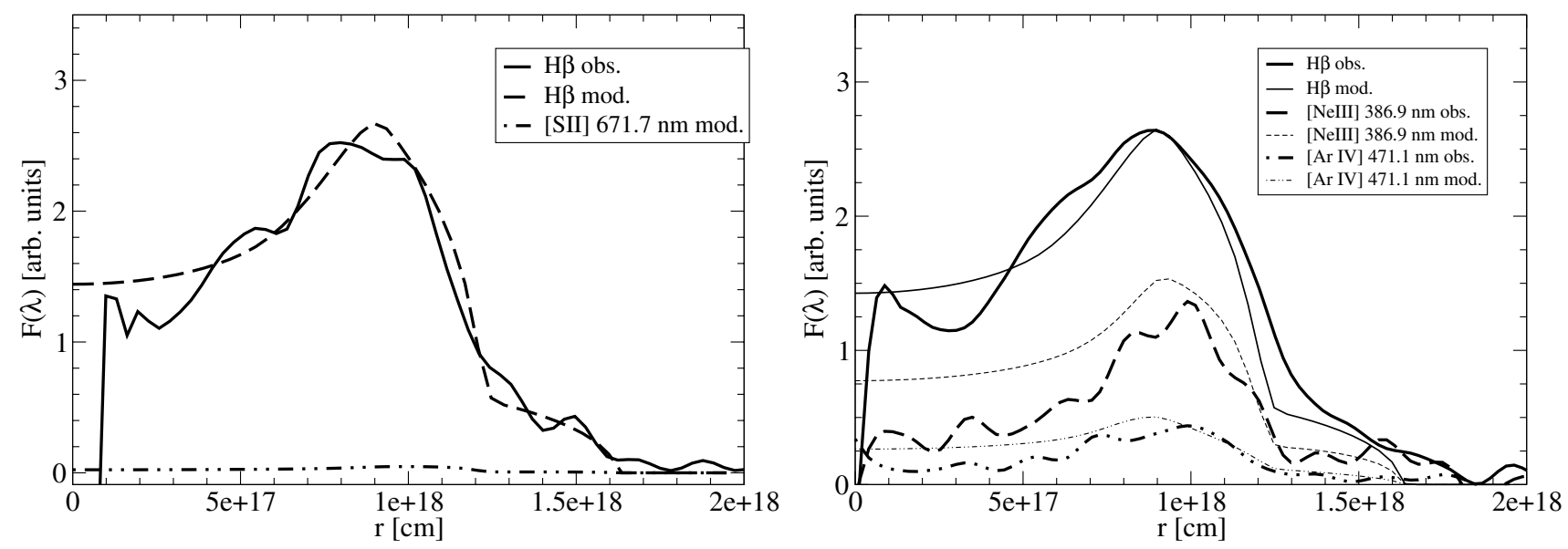

Fig. 2. Comparison of observation and model for several lines of A 20 (same as in Fig. 1).

compared with the observations to determine the best fit. The average electron temperature given by CLOUDY was compared with the values derived earlier from the [OIII] lines. Because of the weakness of the $436.3 \mathrm{~nm}$ line a spatially resolved study was not possible here.

The objects A 15 and MeWe 1-3 showed enhancement in the southern regions, especially in [OIII]. This might be due to interaction with the interstellar medium. Thus these regions where not taken into account for the model.

\subsection{A 20 (GPN G214.96+07.81)}

A 20 is the only one of these objects that has been observed with both telescopes, NTT and ESO $3.60 \mathrm{~m}$. The advantage of the doubled observation is that the slit direction was northsouth at the NTT and east-west at the ESO $3.60 \mathrm{~m}$ telescope and thus we were able to rule out systematic effects in the data reduction and due to the assumption of spherical symmetry.

Figures 1 and 2 show the result of the modelling. The model was optimized with respect to the NTT spectra. In the eastwest direction the HeII line is slightly stronger than in the north-south direction but both observed spectra agree fairly well with the model. Just in the very inner part the model displays an emissivity, especially of the [OIII] line, that is too high compared with the observations. This deficiency is, although uncertain, most likely due to deviations from the spherical symmetry assumed in the CLOUDY code. The modelled [SII] and [NII] lines are far below the limit of detection, which agrees with the observations. EFOSC observations do not cover the wavelength range where the [NII] and [SII] lines are located, but they do cover the line of [NeIII] at $\lambda=386.9 \mathrm{~nm}$ and of [ArIV] at $\lambda=471.1 \mathrm{~nm}$. The [ArIV] line is very faint and the [NeIII] line is at the blue limit of the spectrograph, where the efficiency of the CCD and the transparency of the spectrograph are not good. Thus their error is higher than that of the other lines.

Figure 3 shows the density profile of A 20. The heavyparticle density is mostly that of hydrogen and helium. Other elements contribute less than $2 \times 10^{-4}$. The electron density, which exceeds the hydrogen density twice as much as the heavy particle density, indicates that helium is mostly doubly ionized, which is confirmed in Fig. 4. 


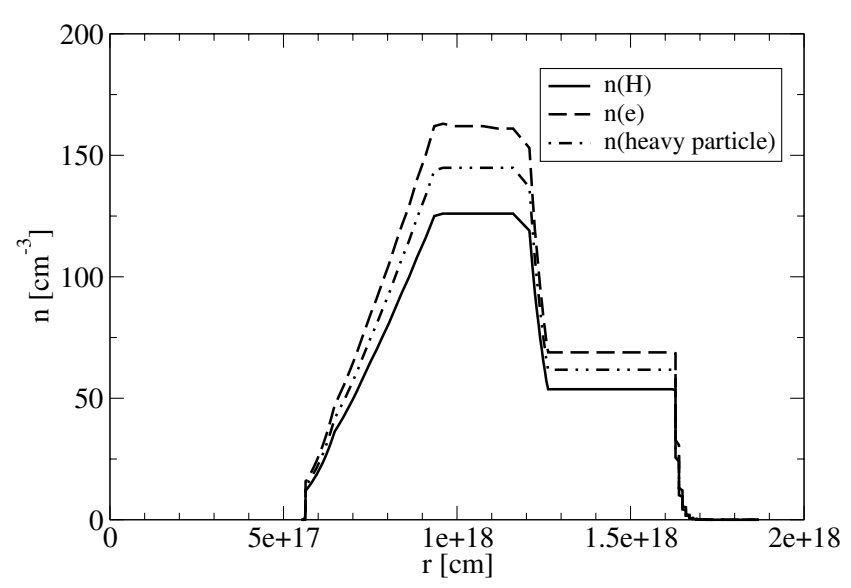

Fig. 3. Density profile of A 20. The hydrogen and electron density have been computed by CLOUDY. The heavy-particle density is calculated from the hydrogen density and the chemical composition.

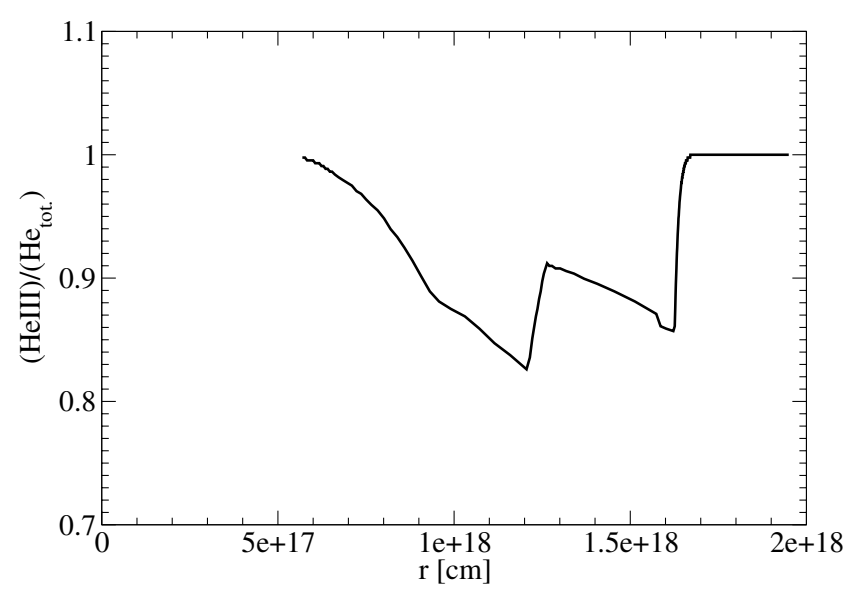

Fig. 4. The fraction of doubly ionized helium as a function of radius. As can be seen helium is mostly doubly ionized, as was expected considering the high HeII $468.6 \mathrm{~nm}$ intensity.

The chemical composition (Table 5) clearly shows an underabundance of heavy elements by a factor of 10 . This makes it most likely that the PN is a member of the galactic thick disk population. The total absence of [SII] and [NII] lines can be explain just in part by underabundance of these elements. These elements may also be mainly at a higher ionization level. The overabundance of $\mathrm{He}$ is typical for $\mathrm{PNe}$ after the dredge-up processes during the RGB/AGB phase.

The halo of the nebula is clearly highly ionized. It is not a recombination halo as predicted by $1 \mathrm{D}$ hydrodynamical simulations.

\subsection{A 15 (GPN G233.53-16.31)}

Figure 5 shows the surface brightness distributions for several lines of A 15. There is hardly any decrease of the intensity towards the center of the nebula, contrary to A 20. This indicates that the inner part of the nebula is not empty, as in A 20, but filled, as can be seen in Fig. 6. The intensity of [SII] and [NII] is again, in agreement with the observations, below the threshold of detection. Also here the HeII line looks like $\mathrm{H} \beta$. Also
Table 5. Chemical composition of A 20. The abundances of each element $Z$ are given in $\log \frac{[Z]}{[\mathrm{H}]}$ and the comparison with the solar abundances (Grevesse et al. 1996) is given in dex.

\begin{tabular}{lccc}
\hline & Abundance & $\begin{array}{c}\text { Abundance compared } \\
\text { to solar abundance }\end{array}$ & $\begin{array}{c}\text { Dominating } \\
\text { ionization level }\end{array}$ \\
\hline \hline $\mathrm{He}$ & -0.825 & 0.175 & $\mathrm{He}$ III \\
$\mathrm{C}$ & -4.45 & -1.00 & $\mathrm{C}$ IV \\
$\mathrm{N}$ & -5.00 & -0.97 & $\mathrm{~N}$ IV \\
$\mathrm{O}$ & -4.10 & -0.97 & $\mathrm{O}$ IV \\
$\mathrm{Ne}$ & -4.824 & -0.90 & $\mathrm{Ne}$ IV \\
$\mathrm{Mg}$ & -5.523 & -1.10 & $\mathrm{Mg}$ III \\
$\mathrm{Si}$ & -5.523 & -1.07 & $\mathrm{Si} \mathrm{III}$ \\
$\mathrm{S}$ & -5.824 & -1.15 & $\mathrm{~S} \mathrm{IV}$ \\
$\mathrm{Ar}$ & -5.80 & -0.32 & $\mathrm{Ar}$ IV \\
\hline
\end{tabular}

Table 6. Chemical composition of A 15 (same as in Table 5).

\begin{tabular}{lccc}
\hline \hline & Abundance & $\begin{array}{c}\text { Abundance compared } \\
\text { to solar abundance }\end{array}$ & $\begin{array}{c}\text { Dominating } \\
\text { ionization level }\end{array}$ \\
\hline $\mathrm{He}$ & -0.82 & 0.18 & $\mathrm{He} \mathrm{III}$ \\
$\mathrm{C}$ & -4.95 & -1.50 & $\mathrm{C} \mathrm{V}$ \\
$\mathrm{N}$ & -5.03 & -1.0 & $\mathrm{~N} \mathrm{IV}$ \\
$\mathrm{O}$ & -4.60 & -1.47 & $\mathrm{O} \mathrm{IV}$ \\
$\mathrm{Ne}$ & -5.420 & -1.50 & $\mathrm{Ne} \mathrm{IV}$ \\
$\mathrm{Mg}$ & -5.920 & -1.50 & $\mathrm{Mg}$ III \\
$\mathrm{Si}$ & -5.950 & -1.5 & $\mathrm{Si} \mathrm{V}$ \\
$\mathrm{S}$ & -6.17 & -1.5 & $\mathrm{~S} \mathrm{~V}$ \\
$\mathrm{Ar}$ & -6.98 & -1.5 & $\mathrm{Ar} \mathrm{IV}$ \\
\hline
\end{tabular}

note that the $[\mathrm{OIII}]$ line is less prominent than $\mathrm{H} \beta$ and the HeII line.

In the inner part of the nebula the density relates to the radius as $r^{-0.3}$. The main shell is thinner and has a lower density than the one of A 20 and is surrounded by a relatively extended and dense halo. The hydrogen in A 15 is also totaly ionized, as is most of the helium (Fig. 6).

The fraction of heavy elements in this object is even lower than in A 20 and the electron temperature thus is higher as the cooling by the forbidden lines is less effective (see Table 3), which still agrees with the $T_{\mathrm{e}}=23000 \mathrm{~K}$ computed by CLOUDY. Also the dominating ionization levels are slightly higher than those in A 20, especially the one of C, S, and Si. The typical He enrichment is shown by A 15 too. The overabundance of nitrogen with respect to the other heavy elements does not hint at a massive progenitor, but is due to the line being an upper limit.

\subsection{MeWe 1-3 (GPN G308.26+07.79)}

MeWe 1-3 shows a central cavity, though not such a pronounced one as in A 20. It also looks like A 20 in other respects, such as the similarity of the HeII and the $\mathrm{H} \beta$ line, the relative line strength of [OIII] and the lack of any [SII] and [NII] lines. Only there is hardly any halo visible in MeWe 1-3.

Since the emissivity distribution of MeWe 1-3 looks like the one of A 20 their density profile is similar - an empty inner part, and a rapid increase of the density to a plateau, in this case 

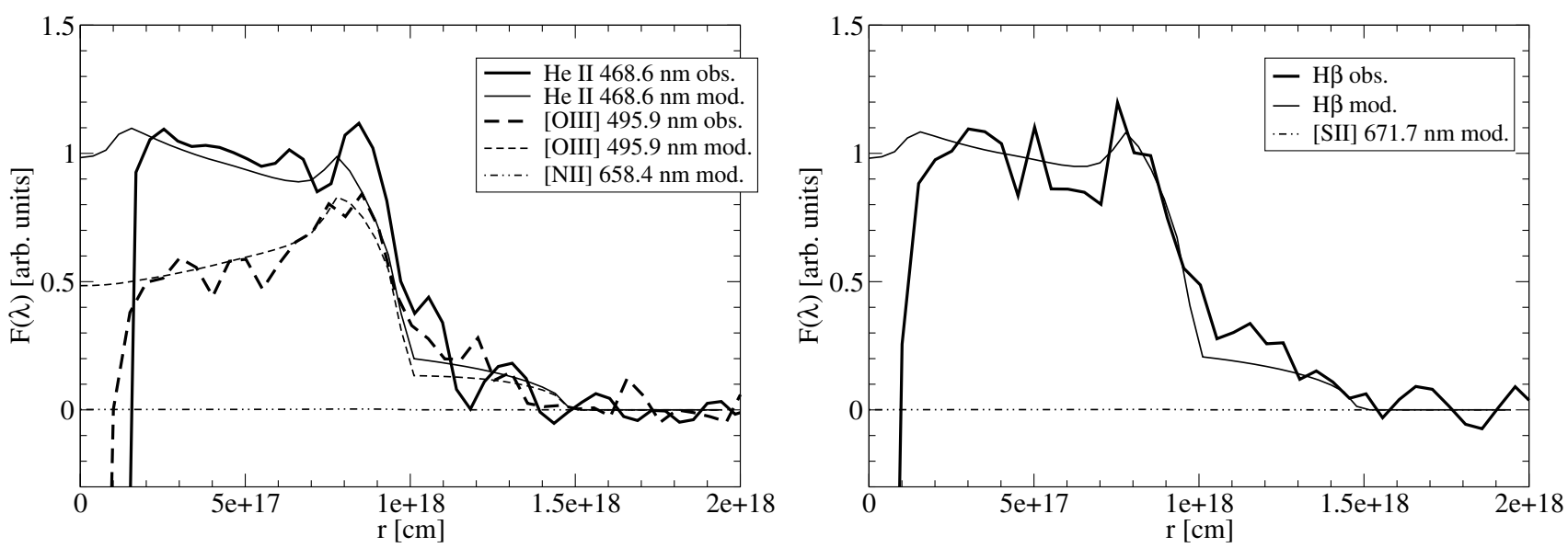

Fig. 5. Comparison of observation and model of several lines of A $15 . \mathrm{H} \alpha$ and [OIII] $(\lambda=500.7 \mathrm{~nm})$ have not been taken into account since they are related to $\mathrm{H} \beta$ and [OIII] $(\lambda=495.9 \mathrm{~nm})$ respectively. The [NII] and the [SII] line were not observable. The innermost "hole" in the data is an artifact of the CSPN subtraction/overlay.
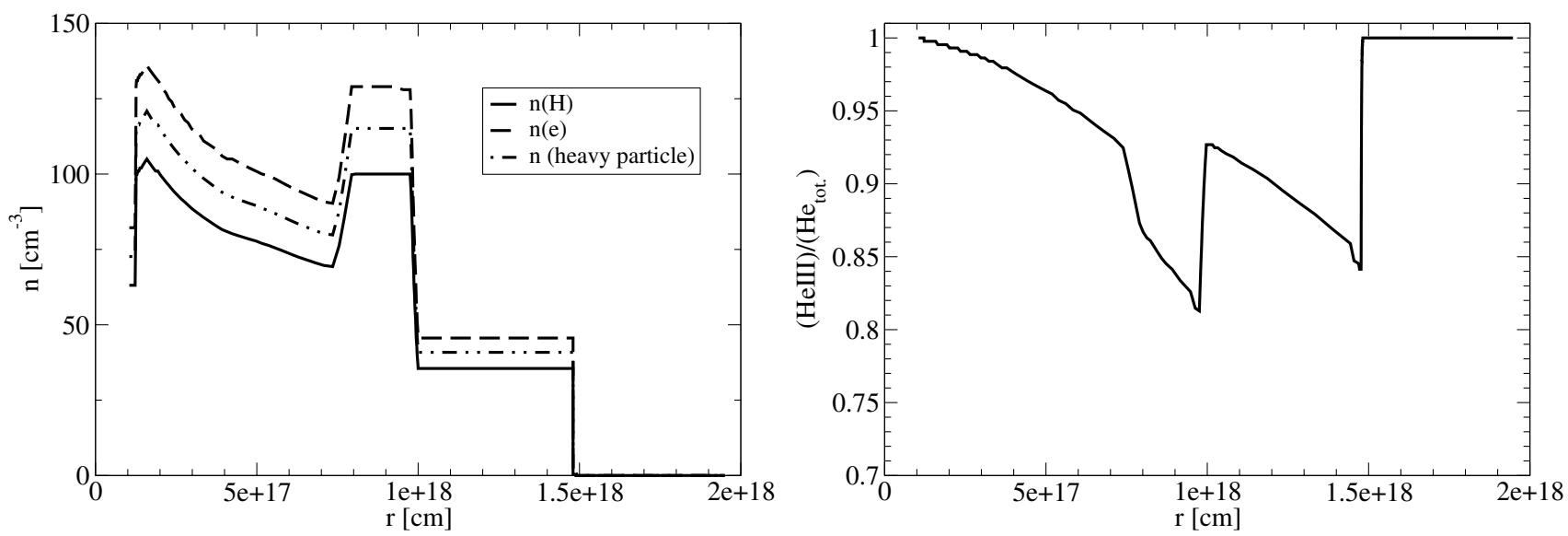

Fig. 6. Density profile and fraction of doubly ionized helium of A15. Description as in Figs. 3 and 4

Table 7. Chemical composition of PN MeWe 1-3 (same as in Table 5).

\begin{tabular}{lccc}
\hline \hline & Abundance & $\begin{array}{c}\text { Abundance compared } \\
\text { to solar abundance }\end{array}$ & $\begin{array}{c}\text { Dominating } \\
\text { ionization level }\end{array}$ \\
\hline $\mathrm{He}$ & -0.85 & 0.15 & $\mathrm{He}$ III \\
$\mathrm{C}$ & -4.45 & -1.0 & $\mathrm{C} \mathrm{IV}$ \\
$\mathrm{N}$ & -5.03 & -1.0 & $\mathrm{~N} \mathrm{IV}$ \\
$\mathrm{O}$ & -4.13 & -1.0 & $\mathrm{O}$ IV \\
$\mathrm{Ne}$ & -5.03 & -1.11 & $\mathrm{Ne} \mathrm{IV}$ \\
$\mathrm{Mg}$ & -5.42 & -1.0 & $\mathrm{Mg} \mathrm{III}$ \\
$\mathrm{Si}$ & -5.45 & -1.0 & $\mathrm{Si} \mathrm{V}$ \\
$\mathrm{S}$ & -5.67 & -1.0 & $\mathrm{~S} \mathrm{~V}$ \\
$\mathrm{Ar}$ & -6.48 & -1.0 & $\mathrm{Ar} \mathrm{IV}$ \\
\hline
\end{tabular}

at $100 \mathrm{H} \mathrm{cm}^{-3}$. Only the thinness of the halo of only $0.013 \mathrm{pc}$ is different. Almost all the nebular hydrogen and helium has been stripped of electrons too.

Also the abundances of the elements are very similar to those of A 20. But the average ionization stage is closer to that of A 15 . The electron temperature $(15800 \mathrm{~K}$ observation, $22500 \mathrm{~K}$ model) is intermediate between those of the two
Table 8. Obtained basic data of the PNe modeled.

\begin{tabular}{lcccc}
\hline \hline Name & $T_{\text {eff }}[\mathrm{kK}]$ & $L\left[L_{\odot}\right]$ & Radius [pc] & Dist. [kpc] \\
\hline A 20 & 140 & 3000 & 0.40 & 2.35 \\
A 15 & 120 & 4200 & 0.32 & 4.01 \\
MeWe 1-3 & 110 & 4500 & 0.21 & 3.95 \\
\hline
\end{tabular}

other nebulae, despite the lower $T_{\text {eff }}$ of its central star. This effect can be explained by the higher luminosity of the CSPN of MeWe 1-3 and the smaller radius of the nebula itself.

Since the errors of the properties of the objects given in the literature are relatively large, especially for the luminosity and the distance, we varied these properties within the ranges of error. The obtained data are given in Table 8 .

It seems that the temperature and thus the luminosity of the CSPN of A 20 are underestimated. The uncertain distances of A 20 and MeWe 1-3 are always smaller in the models than in the literature, but still within the error. 

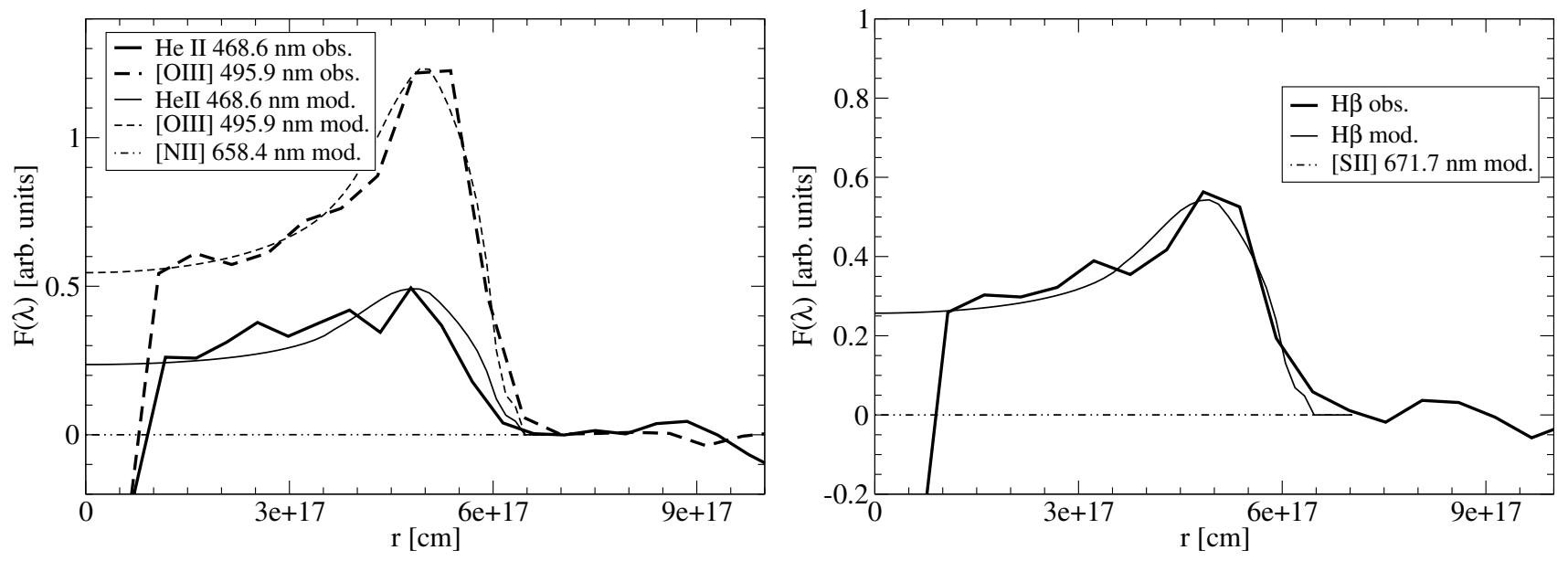

Fig. 7. Comparison of observation and model of several lines of MeWe 1-3 (description see Fig. 5).
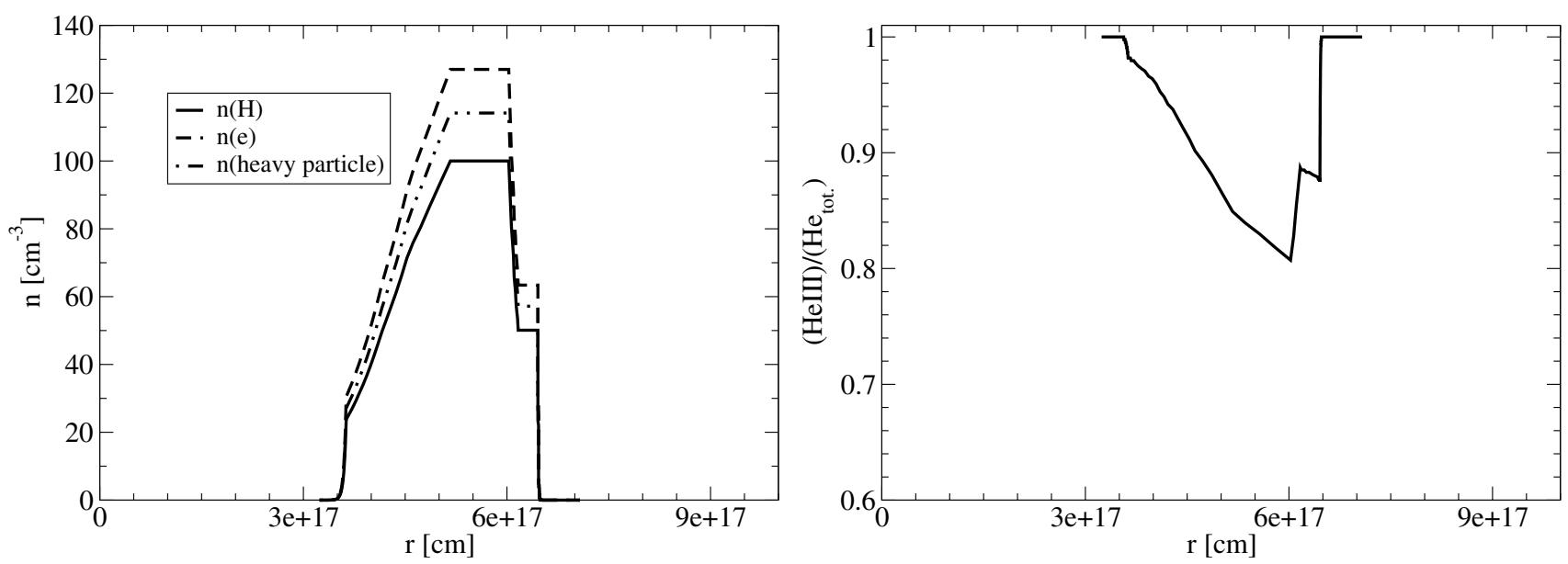

Fig. 8. Density profile (left) and fraction of doubly ionized helium of MeWe 1-3.

\section{Discussion and conclusion}

The metallicities of all three PNe investigated are lower by a factor of 10 to 30 than the solar abundances, only helium is slightly more abundant more present than in the Sun. Soker (2002) already reported an under-abundance of metals in circular PNe.

From the galactic coordinates and the distances of the PNe one can easily verify that the height above the galactic plane $(z)$ of these nebulae is comparatively large (Fig. 9).

The nebulae investigated are located at a $z$ of $320 \mathrm{pc}$, -1127 pc and 535 pc for A 20, A 15 and MeWe 1-3 respectively. To compare these values with others the PNe catalogued by Acker et al. (1992) were taken as a representative sample for all PNe. Half of these planetary nebulae are located below a $z$ of $162 \mathrm{pc}$. Their average $z$ is $288 \mathrm{pc}$. The large $z$ of the three objects investigated indicates that they are so-called thick disk objects and that their progenitors also were low-abundance objects.

The high electron temperature of the nebulae is also linked to the low metallicity (Stasinska 1978). As Frank \& Mellema (1994b) showed, one important cooling mechanisms in ionized gas is the radiation emitted by collisionally excited lines. The most important role is played by the [NII] lines in

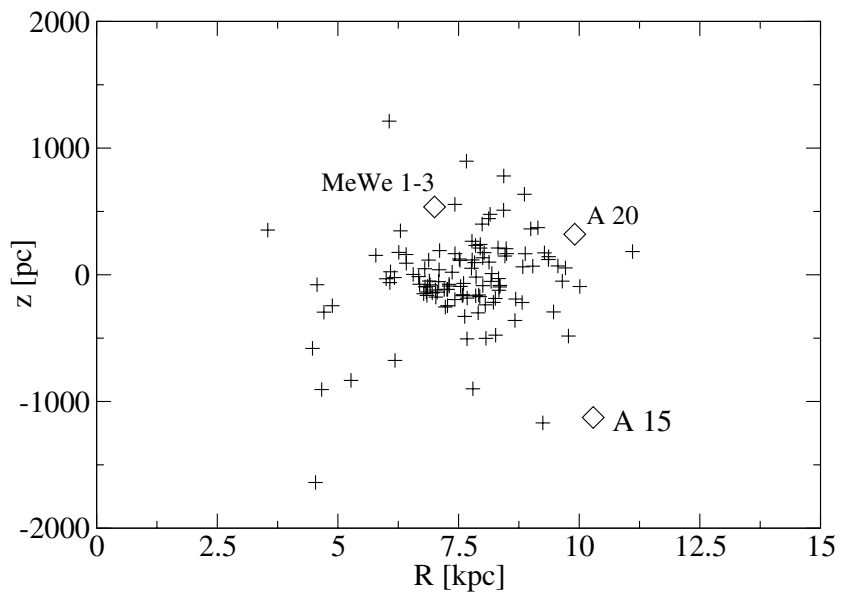

Fig. 9. The Galactic height $z$ versus the radius from the Galactic center. The radius of the sun was assumed to be $8 \mathrm{kpc}$. +: The PNe catalogued by Acker et al. (1992); $\diamond$ : the PNe A 20, A 15 and MeWe 1-3.

low-excitation nebulae and the [OIII] lines in high-excitation ones. Since compared to other PNe no [NII] and less intensive [OIII] lines are detected, a higher temperature is feasible. 
As mentioned above, a filling factor $(\varepsilon)$ of 0.1 was adopted. Since radio data are available for A 20 and A 15 (Condon \& Kaplan 1998) it was possible to check this assumption. Adopting an optically thin nebula in the radio regime and therefore $I_{v} \propto v^{-0.1}$ allows us to compute the radio flux at $5 \mathrm{GHz}$ from the $1.4 \mathrm{GHz}$ data and thus the total ionized mass following Cahn et al. (1992).

$M_{\text {ion. }}=\sqrt{2.266 \times 10^{-21} \cdot D^{5} \cdot \theta^{3} \cdot F_{5 \mathrm{GHz}}}$

where $D$ is the distance in pc, $\theta$ the angular radius in arcseconds and $\mathrm{F}_{5 \mathrm{GHz}}$ the radio flux at $5 \mathrm{GHz}$ in $\mathrm{Jy}$. We compared this mass with the one of our model, computed by

$M=4 \pi \mu \int_{0}^{+\infty} n_{\mathrm{hp}}(r) r^{2} \mathrm{~d} r$

$\mu$ is the mean atomic weight and $n_{\mathrm{hp}}(\mathrm{r})$ the heavy-particle density as a function of the radius. This gives $\varepsilon$. Although the error of the obtained filling factor is quite high it is clearly smaller than 0.3. Using the sample and the formula of Mallik $\&$ Peimbert (1988) we even obtain a value below 0.05 . The formula of Boffi \& Stanghellini (1994) gives a value of 0.096. But these sample shows no PN with a radius above $0.2 \mathrm{pc}$ and a filling factor below 0.1. Tests with CLOUDY show that the nebulae are always optically thin for UV radiation up to $\varepsilon=0.5$.

Further calculations showed that even stars with luminosities down to $160 L_{\odot}$ are able to ionize most of the hydrogen and thus a recombination of the shell seems to be impossible. This is in clear contradiction to the predictions of the 1D hydrodynamical simulations (e.g. Schönberner \& Steffen 2002).

The luminosities of the CSPN found from the investigation of the PN shells here are about the same as those found from spectroscopy using NLTE models. Our temperatures are - still within the errors - slightly higher than those from the CSPN spectra. As shown by Lechner \& Kimeswenger (2004) and Emprechtinger et al. (2004) these parameters are very sensitive to the line ratios of the highly ionized species. We thus suggest that this effect is real. The distances $D_{\text {CSPN }}$ have to be significantly lower than those found in previous investigations.

Acknowledgements. This research was supported by the "Zentrum für Luft- und Raumfahrt" (DLR) under grant 50 OR 0201 (TR). We thank the referee R. Szczerba for the valuable comments improving the original manuscript.

\section{References}

Abell, G. O. 1955, PASP, 67, 258

Acker, A., Ochsenbein, F., Stenholm B., et al. 1992, Strasbourg-ESO Catalogue of Planetary Nebulae

Armsdorfer, B., Kimeswenger, S., \& Rauch, T. 2002, Rev. Mex. Asrton. Astrofis. Ser. Conf., 12, 180

Blöcker, T. 1995, A\&A, 299, 755

Boffi, F. R., \& Stanghellini, L. 1994, A\&A, 284, 248

Cahn, J. H., Kaler, J. B., \& Stanghellini, L. 1992, A\&AS, 94, 399

Condon, J. J., \& Kaplan, D. L. 1998, ApJS, 117, 361

Dwarkadas, V., \& Balick, B. 1998, ApJ, 497, 267

Emprechtinger, M., Forveille, T., \& Kimeswenger, S. 2004, A\&A, 423, 1017

Ferland, G. 1996, A Brief Introduction to Cloudy 90.05, Univ. Kentucky, Department of Physics and Astronomy, Internal Report

Frank, A., \& Mellema, G. 1994a, ApJ, 430, 800

Frank, A., \& Mellema, G. 1994b, A\&A, 289, 937

Grevesse, N., Noels, A., \& Sauval, A. J. 1996, ASP Conf. Ser., 99, 117

Hamuy, M., Walker, A. R., Suntzeff, N. B., et al. 1992, PASP, 106, 566

Heber, U., Hunger, K., Jonas, G., \& Kudritzki, R. P. 1984, A\&A, 130, 119

Kimeswenger, S. 2001, Rev. Mex. Astron. Astrofis., 37, 115

Kwok, S., Purton, C. R., \& Fitzgerald, P. M. 1978, ApJ, 219, 125

Lechner, M. F. M., \& Kimeswenger, S. 2004, A\&A, 426, 145

Mallik, D. C. V., \& Peimbert, M. 1988, Rev. Mex. Asrton. Astrofis., 16,111

McCarthy, J. K., Mendez, R. H., \& Kudritzki, R. P. 1997, IAUS, 180, 120

Melmer, D., \& Weinberger, R. 1990, MNRAS, 243, 236

Mendez, R. H., Kudritzki, R. P., Herrero, A., Husfeld, D., \& Groth, H. G. 1988, A\&A, 190, 113

Rauch, T. 1997, A\&A, 320, 237

Rauch, T. 2003, A\&A, 403, 709

Rauch, T. 2004 http://astro.uni-tuebingen.de/ rauch/

Rauch, T., Köppen, J., Napiwotzki, R., \& Werner, K. 1999, A\&A, 347, 169

Saurer, W., Werner, K., \& Weinberger, R. 1997, A\&A, 328, 598

Savage, B. D., \& Mathis, J. S. 1979, ARA\&A, 17, 73

Schönberner, D., \& Steffen, M. 2002, Rev. Mex. Astron. Astrofis., 12, 144

Soker, N. 1997, ApJS, 112, 487

Soker, N. 2002, A\&A, 386, 885

Stasinska, G. 1978, A\&AS, 32, 429

Steffen, M., Szczerba, R., \& Schönberner, D. 1998, A\&A, 337, 149

Tylenda, R., Acker, A., Stenholm, B., \& Koeppen, J. 1992, A\&AS, 95, 337

Tylenda, R., Sidmiak, N., Gorny, S. K., Corradi, R. L. M., \& Schwarz, H. E. 2003, A\&A, 405, 627 